\title{
Enfermedad renal crónica avanzada en el contexto de polineuropatía amiloidótica familiar tipo I
}

\author{
Daniel Lanzas Martín* - Ignacio Manuel García Tobías* - Elena Barranco Justicia**
}

* Sociedade Portuguesa de Diálise. Grupo Diaverum. Amadora (Portugal) - ** CHD Nefrolinares (Jaén)

\section{Introducción}

Los enfermos con Polineuropatía Amiloidótica Familiar (PAF) tipo I o Enfermedad de Andrade constituyen una minoría con una enfermedad poco frecuente que puede afectar a muchos miembros de una misma familia por su carácter hereditario e inicio insidioso y tardío. Portugal ${ }^{1}$, Suecia², Japón ${ }^{3}$, Brasil, Estados Unidos $^{4}$, Mallorca ${ }^{5}$ entre otros, son regiones donde se ha documentado la enfermedad o sus variantes. El depósito de la sustancia amiloide en el espacio extracelular de los tejidos puede causar su disfunción ${ }^{6}$. De acuerdo con Lobato ${ }^{7}$, la enfermedad, está asociada a una mutación genética de la proteína transtirretina (TTR), sintetizada en el hígado, donde se sustituye una valina por una metionina en la posición 30 (Val30Met).

De inicio periférico y con carácter disto-proximal, la PAF cursa con una polineuropatía inicialmente sensitiva y más tarde motora y autonómica. También están descritas alteraciones gastrointestinales, cardiacas, renales, oculares y de la integridad cutánea ${ }^{1,4,7}$. El trasplante hepático ${ }^{7,8-10}$, es la única alternativa eficaz de tratamiento. En los enfermos con la variante Val30Met, interrumpe la evolución de la enfermedad y se inician lentas mejorías?.

A nivel renal, se evidencia el deposito de la sustancia amiloide desde una fase precoz, estando presente en todos los enfermos ${ }^{11,12}$. El grado de afectación renal depende en gran medida de la cantidad de deposito amiloide $^{10}$. La inclusión de estos enfermos en un programa de hemodiálisis (HD) regular implica conocer bien la enfermedad de base, para prevenir o minimi-

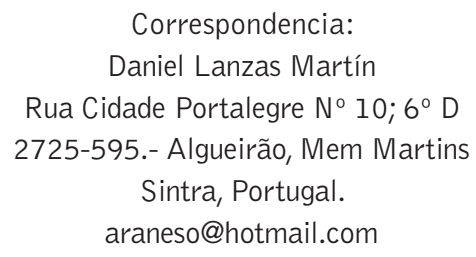

Correspondencia:

Daniel Lanzas Martín

Rua Cidade Portalegre $N^{\circ} 10 ; 6^{\circ} \mathrm{D}$

2725-595.- Algueirão, Mem Martins

Sintra, Portugal.

araneso@hotmail.com

zar los problemas asociados a ésta y los relacionados con el síndrome urémico y el tratamiento con HD. La Diálisis Peritoneal (DP) no está recomendada debido a las alteraciones gastrointestinales y a las limitaciones motoras que estos enfermos desarrollan10. Nuestro objetivo al presentar este caso clínico es profundizar en el estudio y observación de la PAF en pacientes en HD.

Mediante un instrumento de valoración de enfermería, elaborado por los autores, se describe un caso de PAF en HD. Se obtiene un plan de cuidados donde cada uno de ellos se corresponde con resultados y actividades de enfermería, mensurables y clasificadas por patrones funcionales de salud entre marzo y mayo de 2008 en un centro de HD del grupo Diaverum. Se realizó la recogida a partir de la segunda hora de tratamiento (después del bocadillo). Los datos provenientes de la exploración física así como los relativos a la sexualidad y creencias se realizaron en privado.

\section{Resumen historia clínica y valoración de enfermería} El caso que presentamos es el de una señora de 50 años, antecedentes familiares de neuropatía, sin diabetes asociada. La enfermedad se inició hace unos 7 años (1998), con molestias neuropáticas progresivas de predominio distal. Se estableció el diagnóstico de PAF basado en la historia familiar, la clínica, pruebas de laboratorio y biopsia del nervio safeno. Inició diálisis el 17 de junio de 2005, con problemas asociados al acceso venoso; se realizó una fístula arteriovenosa el 3 agosto de 2005.

Madre de 6 hijos, solo uno de ellos ha sido estudiado por presentar signos de perdida de sensibilidad. Vive con su marido y dos hijas y recibe el tratamiento de HD desde hace tres años en un centro de diálisis en las proximidades de su área de residencia. Presenta función renal residual. No consume sustancias tóxicas. Se ve a sí misma como una persona comunicativa aunque se estresa con facilidad. El traslado desde casa al centro de diálisis en la ambulancia le provoca estrés. 
Su peso es de $37 \mathrm{~kg}$, talla es de $148 \mathrm{~cm}$, IMC de 16,89 . La ganancia de peso interdiálisis media es de 1.100 gramos sin oscilaciones en el peso seco.

Exploración física:

- Tiene amputado el $2^{\circ}, 4^{\circ}$ y $5^{\circ}$ dedo de la mano izquierda

- Ausencia de piezas dentales, sin prótesis dentaria

- Presenta pérdida de masa muscular y grasa moderada que limita su capacidad funcional

- Presenta incontinencia fecal por lo que usa de pañal. Su patrón de eliminación habitual es de una a tres deposiciones diarias.

- La sensibilidad, el tono y la fuerza muscular de los miembros inferiores están muy deteriorados no sintiendo nada a partir de las rodillas. Tiene miedo a caer si intenta deambular sin ayuda.

En relación a las actividades de la vida diaria, sabe leer y escribir, muestra dependencia en aquellas que hacen referencia al mantenimiento del hogar y es independiente en el autocuidado. Padece de insomnio de conciliación, que lo achaca al estrés y a la HD para el que no sigue ningún tratamiento farmacológico o no farmacológico. Vive el dolor como una experiencia intermitente, intensa, no irradiada y punzante, sobretodo en los pies, con una frecuencia no definida; lo trata con medidas medicamentosas y no medicamentosas. Reconoce cambios en su cuerpo y reconoce que representan un problema de dependencia. Tiene fe en Dios y fuerza de vivir, el hecho de nunca haber perdido la esperanza; le gusta ir a la Iglesia

El núcleo familiar le presta mucho apoyo. Un hijo murió hace unos seis años, refiere haber superado el proceso de luto y aceptado la perdida de su hijo. Es buena vecina, teniendo amigas dentro del vecindario. Confiesa disfunción sexual desde hace mucho y que en lo que respecta a las relaciones sexuales, el marido la comprende y la acepta.

Tratamiento con hemodiálisis. Realiza HD de alto flujo (Polyflux ${ }^{\circledR} 140 \mathrm{H}$ ) con una duración de tres horas, los martes, jueves y sábado a las siete y media de la mañana. Tiene una FAV autóloga en la fosa antecubital izquierda que se punciona con agujas 15G en ambos sentidos. El flujo de sangre estipulado es de $350 \mathrm{ml} / \mathrm{min}$ y el de líquido de diálisis de $700 \mathrm{ml} /$ min. La anticoagulación se realizada con heparina sódica.
Las etiquetas diagnósticas contempladas en el Plan de Cuidados fueron las siguientes:

- Dolor crónico

- Temor

- Deterioro del patrón del sueño

- Intolerancia a la actividad

- Desequilibrio nutricional por defecto

- Conocimientos deficientes

- Incontinencia fecal

- Trastorno de la imagen corporal

- Trastorno de la percepción sensorial (visual y táctil)

- Riesgo de deterioro de la integridad cutánea

- Riesgo de Infección

- Diarrea

A modo de ejemplo el anexo 1 recoge el desarrollo de la etiqueta diagnóstica "Dolor crónico" con sus resultados e indicadores

\section{Discusión}

En la atención de los enfermos renales en general, es fundamental e indispensable conocer su enfermedad de base con el objetivo de proporcionar cuidados específicos e individualizados. Todos ellos experimentan un cambio de vida radical que incluye alteraciones en el aspecto físico, psíquico y social ${ }^{13}$. Los enfermos con PAF y enfermedad renal no escapan a esta regla y están poco estudiados por la enfermería nefrológica en nuestro medio. Por ello, el presentar un caso con PAF en HD, permite dar a conocer la enfermedad a la comunidad de enfermería de nefrología. La propuesta de plan de cuidados que aquí se sugiere hace pensar que con las mismas intervenciones, podemos solucionar total o parcialmente más de un diagnósti$\mathrm{Co}^{13}$, considerando que los planes de cuidados no sean absolutos ya que la elaboración de un plan de cuidados, es un método sistematizado que permite unificar criterios que encaminen la práctica enfermera hacia la consecución de objetivos (independientes o de colaboración), de forma eficiente ${ }^{14,15}$.

La puesta en práctica de este plan de cuidados ha permitido detectar problemas reales y potenciales de la paciente y la familia. De esta forma ha sido posible enfocar la actuación de enfermería para un mejor aprovechamiento por parte de la paciente y 


\begin{tabular}{|c|c|}
\hline \multicolumn{2}{|c|}{ Etiqueta diagnóstica: Dolor crónico } \\
\hline Relacionado con & Manifestado por \\
\hline Dolor óseo, dolor al defecar & Expresiones verbales de dolor vivido intensamente y lloro por causa del dolor \\
\hline \multicolumn{2}{|c|}{ Escala: 1.- Nunca demostrado; 2.- Raramente demostrado;3.- En ocasiones demostrado; 4.- Con frecuencia demostrado; 5.- Constantemente demostrado } \\
\hline Resultados (NOC): indicadores & Intervenciones (NIC): actividades \\
\hline 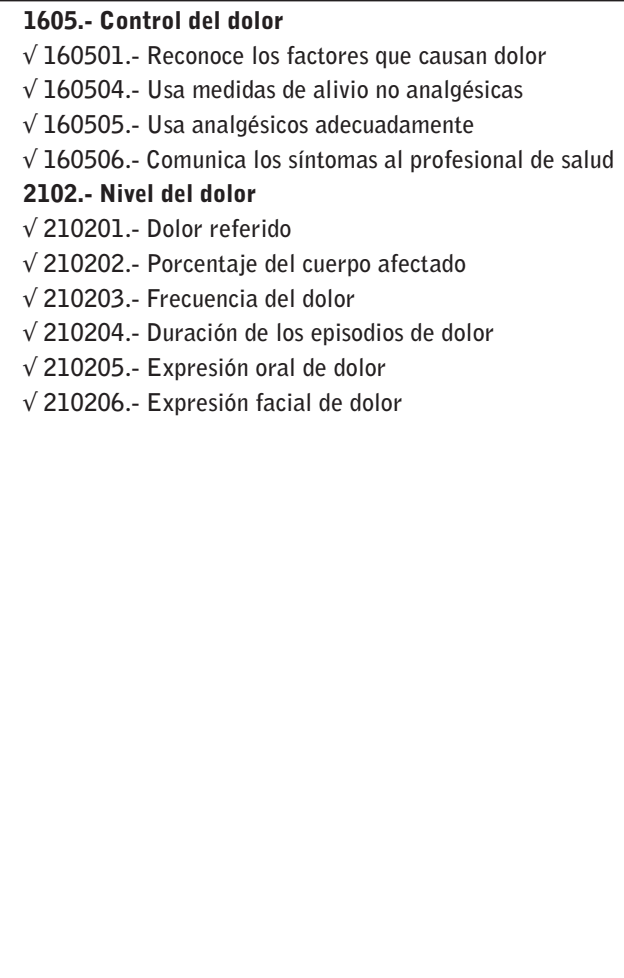 & $\begin{array}{l}\text { 1400.- Control del dolor } \\
\sqrt{ } \text { 140001.- Realizar una valoración completa del dolor, que incluya el local, } \\
\text { las características, el inicio, duración, frecuencia, calidad, intensidad y } \\
\text { gravedad del dolor y los posibles factores desencadenantes } \\
\sqrt{ } \text { 140002.- Observar indicadores no verbales de incomodidad } \\
\sqrt{ } \text { 140003.- Asegurar que la paciente recibe cuidados precisos de analgesia } \\
\sqrt{ } \text { 140007.- Determinar el impacto de la experiencia del dolor sobre la calidad } \\
\text { de vida (sueño, apetito, actividad, relaciones...) } \\
\sqrt{ } \text { 140008.- Investigar con la paciente los factores que alivian o desencadenan } \\
\text { el dolor. } \\
\sqrt{ } \text { 140024.- Orientar sobre métodos farmacológicos de alivio del dolor. } \\
\sqrt{ } \text { 140025.- Alentar a la paciente a utilizar el medicamento adecuado para el } \\
\text { alivio del dolor, con uso a los medicamentos prescritos } \\
\text { 2210.- Administración de analgésicos (durante el tratamiento con HD) } \\
\sqrt{ } 221001 \text {.- Determinar el local, las características, la calidad y la intensidad } \\
\text { del dolor antes de administrar el medicamento } \\
\sqrt{ } 221002 \text {.- Verificar la prescripción medica, la dosis, la frecuencia, la vía de } \\
\text { administración del medicamento prescrito } \\
\sqrt{ } 221004-\text { Valorar la capacidad de la paciente para participar en la selección } \\
\text { del analgésico. } \\
\sqrt{ } 221013 \text {.- Establecer expectativas positivas en cuanto a la eficacia de los } \\
\text { analgésicos, de modo a optimizar su respuesta. } \\
6482 .- \text { Control del ambiente: Confort (comodidad) } \\
\sqrt{ } 648203 \text {.- Determinar fuentes de incomodidad tales como temperatura de la } \\
\text { sala muy fría o caliente, arrugas en el pañal o en la ropa del sillón, posición } \\
\text { confortable. } \\
\sqrt{ } 648210 \text {.- Controlar el ruido indeseado o excesivo } \\
\sqrt{ } 648212 \text {.- Posicionar a la paciente para facilitar el confort }\end{array}$ \\
\hline
\end{tabular}

con ello mejorar la calidad de los cuidados. Se consiguió implicar a la misma en la promoción del autocuidado, aceptar sus limitaciones y alteraciones en la imagen corporal. Se consiguió mejorar la adherencia terapéutica y dietética mediante la implicación de la familia. Fueron referenciadas para una asociación de solidaridad social que colabora en el estudio de la PAF con un núcleo en Lisboa, para beneficiarse de los servicios que esta entidad ofrece.

\section{Bibliografía}

1. Conceição I. Clínica e Historia Natural da Polineuropatia Amiloidotica Familiar. Sinapse. 2006; 6(1). Supl 1:86-91.

2. Nowak G, Sur 0, Wikström L, Wilczek H, Ericzon $B$. The long-term impact of liver transplantation on kidney function in familiar amyloidotic polyneuropathy patients. Transplant International 2004; 18 (1): 11-115.
3. Misu K, Hattori N, Magamatsu M, Ikeda S, Ando Y, Nakazato M, Takei Y, Hanyu N, Usui Y, Tanaka F, Harada T, Inukai A, Hashizume $Y$, Sobue G. Late-onset familial amyloid polyneuropathy type I (Transthyretin Met30-associated familial amyloid polyneuropathy) unrelated to endemic focus in Japan. Brain 1999; 122:1951-1962.

4. Buades Reinés J, Andreu Serra H. Hereditary familial amyloidosis. Gastroenterol Hepatol 2000; 23: 144-148.

5. Ballesteros Fernández JA. Polineuropatía amiloidótica familiar en Mallorca. Cartas al Editor. Med Clin (Barc) 2004; 122 (5):179-9.

6. Martínez J.M. Amiloidosis. En Farreras Rozman. Medicina Interna. Edición en CD-ROM. 13 Ed. 1127-1130.

7. Lobato L. Classificação das Amiloidoses. Sinapse. 2006; 6(1). Supl 1:68-73. 
8. Lobato L, Beirão I, Silva $M$, Bravo $F$, Silvestre $F$, Guimarães S, Sousa A, Noël LH, Sequeiros J. Familial ATTR amyloidosis: microalbuminuria as a predictor of symptomatic disease and clinical nephropathy. Nephrol Dial Transplant 2003; 18:532538.

9. Munar Qués M. Polineuropatía amiloidótica familiar. Med Clin (Barc) 2003; 121(3):100-1.

10. Lobato L. Nefropatía na Polineuropatía Amiloidótica Familiar. Sinapse. 2006; 6(1). Supl 1:99102.

11. Snanoudj $R$, Durrbach $A$, Gauthier $E$, Adams $D$, Samuel D, Ferlicot $S$, Bedossa P, Prigent A, Bismuth $\mathrm{H}$, Charpentier $\mathrm{B}$. Changes in renal function in patients with familial amyloid polyneuropathy treated with orthotopic liver transplantation. Nephrol Dial Transplant. 2004; 9:1779-1785.

12. Lobato L, Beirão I, Guimarães SM, Droz D, Guimarães S, Grünfeld JP, Noël LH. Familial Amyloid Polyneuropathy Type I (Portuguese): Distribution and Characterization of Renal Amyloid Deposits. American Journal of Kidney Diseases 1998; 31(6):940-946.

13. Torres J, Velasco P, Lla A. Plan de cuidados del paciente en hemodiálisis. En: Libro de comunicaciones del XXXI Congreso de la Sociedad Española de Enfermería Nefrológica; Córdoba 1-4 Octubre 2006. Barcelona: HOSPAL; 2006. p. 235-243.

14. Ruiz MJ, Roman M, Serarols M, Diez de los Rios F. Propuesta de plan de cuidados del paciente con IRC en tratamiento sustitutivo con hemodiálisis. Rev Soc Esp Enferm Nefrol 2001; I trimestre, $n^{\circ}$ 13:72-77.

15. García Palacios, R; Tejuca Marenco, M; Tejuca Marenco, A; Carmona Valiente, MC; Salces Sáez, E; Fuentes Cebada, L. Plan de cuidados personalizado aplicando la taxonomía II NANDA, NOC y NIC. Rev Soc Esp Enferm Nefrol 2002; 18:6972.

\section{BIBLIOGRAFÍA PARA LA CREACIÓN DE LOS PLANES DE CUIDADOS}

- J.C. McCloskey y G.M. Bulechek. Clasificación de Intervenciones de Enfermería (CIE). Nursing Interventions Classification (NIC). Madrid: Ediciones Harcout / Mosby; $3^{a}$ ed. 2002.

- Jonson M, Maas, M, Moorhead S. Clasificación de Resultados de Enfermería (CRE). Nursing Outcomes Classification (NOC). Madrid: Ediciones Harcourt / Mosby; 2a ed. 2002.

- Salces E, Del Campo MC, Carmona MC, Fernández JL, Fernández JJ, Ramírez A. Guía de Diagnósticos Enfermeros del Paciente en Hemodiálisis (Interrelaciones NANDA-NOC-NIC). Ed. Grupo 2 Comunicación. 2005. 